\title{
Dificultades en la implementación de políticas públicas interjurisdiccionales. Análisis del proceso de creación de la Zona Franca Santafesina (Argentina)
}

\author{
Mauricio Moltó \\ Universidad Nacional del Litoral- CONICET, Santa Fe, Argentina \\ Email: mauriciomolto@gmail.com
}

\begin{abstract}
Resumen: Proponemos abordar la complejidad del desarrollo de políticas públicas interjurisdiccionales en sistemas de gobierno federales mediante el análisis de la implementación de la Zona Franca Santafesina, ubicada en la ciudad de Villa Constitución, Provincia de Santa Fe, Argentina. Encontramos este proceso interesante debido a que el diseño de esta política pública implicó la coordinación entre actores públicos de los tres niveles de gobierno y de actores privados, pero particularmente por una serie de contingencias que aparecieron durante la creación de la Zona Franca Santafesina que obstaculizaron su rápida puesta en funciones y complejizaron el esquema de implementación. A su vez, ocasionaron la intervención de actores no contemplados en el esquema original y generaron una serie de situaciones que dilataron sus plazos, dando como resultado un largo proceso que demandó cerca de 26 años.
\end{abstract}

Palabras claves: Implementación de políticas públicas; complejidad de la acción conjunta; zona franca.

\section{Interjurisdictional public policies implementation difficulties. Santafesinian Free Zone's (Argentina) creation process analysis}

\begin{abstract}
: we propouse analyses federal government systems' interjurisdictional public policies complexity by the analysis of the Santafesinian Free Zone implementation in Villa Constitución, Santa Fe province, Argentina. We found this process interesting because of its design implied coordination among multiple government actors from the three levels and some private actors too, but particularly due to a series of contingent events that showed up during the Santafesinian Free Zone's creation process and hindered its quick in place and which increased the original implementation scheme's complexity. In turn, these events caused the intervention of new actors and generated a row of situations which delay the Santafesinian Free Zone's implementation, generating as result a long process which demanded almost 26 years.
\end{abstract}

Keywords: Public policies implementation; complexity of joint action; free zone.

\section{Dificultades na implementação de políticas públicas interjurisdicionais. Análise do processo da criação da Zona Franca Santafesina (Argentina)}

Resumo: Propoemos abordar a complexidade do desenvolvimento de políticas públicas interjurisdicionais nos sistemas de governo federias analisando a implementação da Zona Franca Santafesina, localizada na cidade de Villa Constitución, província de Santa Fe, Argentina. Achamos esse processo interessante porque o disenho dessa política implicou a coordenação entre atores públicos dos três níveis do governo y de atores privados, mas principalmente devido a uma série de contingências que surgiram durante a criação da Zona Franca Santafesina que dificultaram sua criação e tornaram-se mais complexo o esquema de implementação. Por sua vez, causaram a intervenção de actores não contemplados no esquema original e generaram uma série de situações que prolongaram seus prazos, resultando em um longo processo que exigiu quase 26 anos.

Palavras-chave: Implementação de políticas públicas; complexidade da ação conjunta; zona franca. 


\section{Introducción}

En el presente trabajo presentamos parte del análisis desarrollado en nuestra tesis de doctorado. Proponemos abordar la complejidad del desarrollo de políticas públicas interjurisdiccionales en sistemas de gobierno federales mediante el análisis de la implementación de la Zona Franca Santafesina (ZFS), ubicada en la ciudad de Villa Constitución, Provincia de Santa Fe, Argentina. Encontramos este proceso singularmente interesante debido a que el diseño de esta política pública implicó la coordinación entre actores públicos de los tres niveles de gobierno y de actores privados, pero particularmente por una serie de contingencias que aparecieron durante la creación de la ZFS que obstaculizaron su rápida puesta en funciones y complejizaron su esquema de implementación. A su vez, ocasionaron la intervención de actores no contemplados en el esquema original y generaron una serie de situaciones que dilataron sus plazos, dando como resultado un largo proceso que demandó cerca de 26 años. Así, las complicaciones aparecidas llevaron a que las etapas previstas para su creación debieran transitarse dos veces casi por completo.

La motivación de fondo de nuestra investigación está relacionada con la comprensión del funcionamiento del Estado y la articulación entre los actores del gobierno federal, provincial y local durante el desarrollo de políticas públicas, principalmente, en la identificación de los elementos que pueden restarles efectividad y coherencia durante la etapa de implementación.

Tomamos como punto de partida el año 1991, cuando un grupo de actores políticos locales vieron en la posibilidad de creación de una zona franca (ZF) una respuesta viable para hacer frente a una situación económica difícil por la que atravesaba Villa Constitución y su área de influencia. Durante la puesta en práctica de esa solución se toparon con un esquema de relaciones entre los ámbitos local, provincial y nacional, que en la práctica se tornó difícil de llevar adelante. En este sentido, buscamos caracterizar esa dinámica de relaciones a la que Pressman y Wildavsky (1984) llamaron «la complejidad de la acción conjunta», ya que en esta iniciativa intervinieron el Poder Ejecutivo Nacional, a través del Ministerio de Economía; la Administración Nacional de Aduanas; el Poder Ejecutivo Provincial a través del actual Ministerio de la Producción; el Poder Ejecutivo Municipal; el Poder Legislativo Provincial; el Concejo Deliberante de la ciudad de Villa Constitución; un comité de evaluación y selección para llevar adelante la licitación de la ZFS y un comité de vigilancia.

En consideración de esta situación, el objetivo del trabajo es caracterizar algunas de las dificultades que surgen en la coordinación de acciones necesarias para la implementación de políticas públicas interjurisdiccionales en un sistema de gobierno federal. En este sentido, consideramos como hipótesis que una política pública tiende a complejizarse durante su puesta en marcha debido a situaciones contingentes que generan la intervención de nuevos actores, demoras en los plazos y especulaciones que incrementan la probabilidad de fracaso de la iniciativa.

\section{Materiales y métodos}

\section{Marco conceptual}

Cuando mencionamos el proceso de implementación de la ZFS, nos referimos al encadenamiento de acciones que se sucedieron a través de las etapas contempladas en el diseño de esta iniciativa y fueron llevadas adelante por actores políticos y económicos nacionales, provinciales y locales cuya intención era crear y poner en funcionamiento una ZF en la ciudad de Villa Constitución. En este proceso, daremos cuenta de las consecuencias negativas generadas por las tensiones derivadas de los intereses divergentes de algunos de los involucrados y cómo estos condicionaron las articulaciones interinstitucionales.

La pregunta por el proceso y las condiciones de la implementación es amplia, pero básicamente refiere a la indagación sobre las condiciones institucionales y sociales bajo las cuales los promotores de una política pública pueden lograr que los actores sociales implicados pongan en práctica en tiempo y forma las actividades que les fueron asignadas, de las cuales dependen otras y forman una cadena de acontecimientos con resultados parciales que conducen a un resultado general. Una vez tomada la decisión, es necesario que ésta se haga 
operativa. En este proceso, el Estado depende de su organización interna y su capacidad para negociar con actores externos. A su vez, el plano interno puede volverse lento y/o conflictivo, ya que las estructuras orgánicas de los Estados modernos son altamente complejas e implican varios niveles de gobierno y un número relativamente importante de dependencias en cada uno de ellos.

Vemos al Estado desde la perspectiva que Bohoslavsky y Soprano (2010) denominaron como «el Estado desde adentro» y coincidimos con los autores cuando afirman que: 1) el Estado no es un actor «unívoco y autoconsciente»; 2) éste es un espacio "polifónico» donde se cruzan demandas de diferentes grupos de la sociedad; 3) no puede separarse al Estado de quienes lo encarnan, es decir, de aquellos actores que «son el Estado en determinado tiempo y lugar»; 4) la importancia de analizar las tensiones internas entre distintas agencias, niveles de gobierno y actores para el análisis de la acción estatal; y 5) hay que «descentralizar» el estudio del Estado, en el sentido de producir conocimiento sobre realidades subnacionales y locales, y sobre sus diferentes dependencias, agencias y funciones (Bohoslavsky y Soprano, 2010: 23- 28). Consideramos esta perspectiva como punto de partida del análisis, ya que abordamos la acción estatal como resultante de las articulaciones entre los actores involucrados en una política pública. En este sentido, describimos el impacto de las tensiones entre diferentes percepciones, intereses y prioridades de los actores sobre el rumbo que tomó la implementación de la ZFS.

El estudio de la implementación de políticas públicas toma como objeto la ejecución práctica de las decisiones estatales. Puede entenderse como el encadenamiento de las acciones que permiten alcanzar los resultados esperados de una iniciativa. Un proceso complejo donde es común que emerjan problemas que obligan a una redefinición en el diseño, un cambio de rumbo o, directamente, llevan a una iniciativa al fracaso. Refiere a las acciones, arreglos institucionales y políticos, dilaciones, problemas, ajustes, reajustes, etc. que afecten la consecución de las metas trazadas. De este modo, «el análisis de la implementación es el estudio sobre las condiciones bajo las cuales las decisiones de la autoridad conducen efectivamente a los resultados deseados» (Berman, 1993, p.286).

El clásico trabajo de Pressman y Wildavsky (1984) aborda una problemática similar a las inquietudes que motivaron este trabajo. Los autores son unos de los primeros que llamaron la atención sobre las dificultades que implica la acción coordinada entre una variedad de funcionarios y agencias gubernamentales cuando éstas cuentan con diferentes perspectivas y culturas organizacionales. Contra la idea de sentido común de que una mayor cantidad de personas implicadas contribuyen a enriquecer el contenido y a garantizar el éxito de una iniciativa a causa de la comunión de esfuerzos y el mayor consenso que otorga el mayor número de participantes, los autores sostienen que una mayor cantidad de participantes implica una mayor posibilidad de desacuerdos. Bajo la idea de «la complejidad de la acción conjunta», sostienen que mientras más actores participan de una política pública es también mayor la cantidad de transacciones- entre distintas dependencias y al interior de cada una -que son necesarias para su implementación. En consecuencia, mayor es el riesgo de que la iniciativa pueda ser vetada en alguna de esas instancias. El veto no necesariamente constituye una negativa directa, sino que es muy común que se traduzca en una demora indeterminada. Como veremos más adelante, esto fue lo que ocurrió en algunas etapas del proceso de implementación de la ZFS.

Los autores realizan una clasificación de las posibles razones por las cuales pueden surgir desacuerdos entre los participantes implicados en una iniciativa pública (Pressman y Wildavsky, 1984, p.185-188). Mencionan que, aunque exista acuerdo general sobre los objetivos, pueden surgir situaciones que limiten sus posibilidades de éxito. De este modo, (1) puede que un programa tenga sobrados motivos para llevarse adelante, pero genere un conflicto con otras metas correspondientes a alguna de las organizaciones o actores asociados. (2) También, que se prefiera otro programa u otra forma de intervención. (3) Además, los participantes pueden tener compromisos simultáneos que limiten el tiempo dedicado al proyecto. (4) Puede suceder que sea necesario establecer una acción coordinada con organismos o personas necesarios para el éxito de la iniciativa y que estos disientan en la urgencia o en los tiempos establecidos para llevarla adelante. (5) Otra de las razones que conspiran contra la implementación surge cuando existe desacuerdo sobre la división de tareas, el liderazgo o una sospecha sobre las motivaciones de algunos de los líderes o de los actores que pretenden liderar la iniciativa o una parte de ella. (6) También puede haber desacuerdos sobre cuestiones legales o procedimientos técnicos. O bien (7) falta de poder para apoyar e impulsar la iniciativa. 
También nos apoyaremos en algunos de los «juegos de implementación» que describe Bardach (1977), una tipología de dinámicas interactivas entre participantes de una política pública que ocasionan efectos negativos sobre su desarrollo, pudiendo generar desvío de fondos, distorsiones sobre los objetivos y disminución del poder real de los encargados de llevarla adelante debido a la limitación de los apoyos y a distintas estrategias defensivas de algunos participantes.

En nuestro análisis encontramos bien marcada la incidencia de tres elementos de esta tipología: la idea de «apilar» (Bardach, 1977, p. 85-90), es decir, sumar nuevos objetivos cada vez más generales sobre una iniciativa, que distorsiona sus propósitos originales; las falsas adhesiones que recibe una política y que luego no se traducen en un apoyo real (Bardach, 1977, p. 98-103); y una actitud defensiva por parte de actores cuya colaboración era necesaria para llevar adelante la ZFS, que en la práctica funcionó como la inversa del juego de «reputación» que describe Bardach (1977, p.168-172), ya que no se trató de bloquear a actores rivales para que no capitalicen para sí el éxito del proyecto, sino de evitar arriesgar el propio prestigio en una iniciativa cuyo fracaso, de momentos, parecía seguro.

\section{Metodología y fuentes de información}

La principal fuente de información con la que trabajamos son los testimonios de los actores claves que participaron del proceso de implementación. A su vez, para la reconstrucción de los hechos, utilizamos información proveniente de la prensa local y regional, así también como diarios de sesiones parlamentarios, documentos institucionales y normativa nacional y provincial.

De acuerdo con la idea de implementación expresadas en el marco teórico, en primer lugar, consideramos la trayectoria político-burocrática ideal del proyecto ZFS; es decir el recorrido a través de las instancias políticas y administrativas necesarias para su implementación exitosa, un escenario que contempla que todo salió bien desde el principio. Para ello, se recurrió a la legislación nacional y provincial, a resoluciones administrativas y documentos confeccionados por el Instituto Zona Franca Santafesina (IZFS) y del Ente Zona Franca Santafesina (EZFS), a los dictámenes de la Fiscalía de Estado de la Provincia de Santa Fe y a los dictámenes emitidos por la autoridad nacional de aplicación de la ley 24.331.

En segundo lugar, observamos el recorrido que efectivamente siguió la implementación de la ZFS y describimos cómo se resolvieron las distintas instancias necesarias para la implementación, dónde existieron problemas, qué clase de inconvenientes hubo, los actores involucrados y su resolución. Esta tarea implicó la revisión de la reglamentación sobre ZF a nivel nacional y de la ZFS, y de los documentos del archivo del EZFS. Por otro lado, realizamos una reconstrucción de los acontecimientos en base a la prensa local. Analizamos las noticias aparecidas en el periódico local EI Sur y el diario La Capital de la vecina ciudad de Rosario.

La información más rica fue la aportada por los informantes claves. Comenzamos las consultas por las dos puntas del período: primero con el actual presidente del EZFS (en funciones durante el año 2018); segundo con un consultor técnico contratado por el IZFS para asesorar a los responsables de la implementación del proyecto a partir del año 1994 y que participó del proyecto hasta finales de la década de los 90. En base a estos dos contactos iniciales, tuvimos acceso a casi la totalidad del personal político y técnico implicado directamente en la implementación de la ZFS. También tuvimos oportunidad de consultar a legisladores provinciales de Villa Constitución y Rosario, miembros de organizaciones del sector privado, funcionarios del ministerio de la producción provincial (MP), personal de Fiscalía de Estado de la Provincia de Santa Fe, un miembro de la primera empresa concesionaria y otro del concesionario actual.

\section{Resultados}

\section{Contexto de la iniciativa}

En el año 1907, mediante la ley 5.142, el gobierno nacional dispuso la creación de dos ZF. Una en la ciudad de La Plata (provincia de Buenos Aires); la otra, sin especificar, «en un puerto de la provincia de Santa Fe». Hubo 
que esperar hasta el año 1991 para ver novedades, recién cuando por decreto, el Poder Ejecutivo Nacional reglamentó la ley 5.142 en lo que respecta a crear una ZF en la ciudad de La Plata. En ese decreto nada se dijo sobre la otra ZF, la de la Provincia de Santa Fe, pero la decisión de avanzar en la materia por parte del gobierno de Carlos Menem, sumado a que el tema se encontraba en agenda desde fines de los 80, encontró repercusiones entre la dirigencia de la ciudad de Villa Constitución.

Villa Constitución se encuentra ubicada en el extremo sureste de la Provincia de Santa Fe y conforma junto con San Nicolás, Ramallo y otras pequeñas localidades santafesinas y bonaerenses el aglomerado San Nicolás-Villa Constitución (figura 1). En la actualidad, el aglomerado cuenta con un poco más de 200 mil habitantes, de los cuales — si estimamos la población para el año 2019 — 143.215 corresponden a San Nicolás y 50.264 a Villa Constitución. Desde mediados del siglo 20 hasta el presente, la historia del área se encuentra ligada a la industria metalúrgica, principalmente a la Sociedad Mixta Siderúrgica Argentina (SOMISA), creada en 1947, pero cuya inauguración debió esperar hasta 1960; y a la empresa Industria Argentina de Aceros SA (ACINDAR), que instaló su segunda planta en Villa Constitución casi una década más tarde. (Giniger, 2012).

A principios de los años 90, ACINDAR llevó a cabo un proceso de reestructuración de procesos productivos y de métodos de trabajo que dejó el saldo de 954 desvinculaciones, ya sea por despidos, jubilaciones anticipadas o por «retiros voluntarios». La política de reducción de personal propio continuó durante toda la década de los 90 y la empresa pasó de tener 3159 empleados directos en 1990 a 1323 en el año 2001 (Strada, 2017). En paralelo, en el año 1991, en el marco del plan de reforma del Estado impulsado por el gobierno de Menem, comenzó un proceso de «racionalización» de SOMISA a los fines de hacerla más atractiva para su privatización. Esto significó, entre otras cosas, reducir la planta de trabajadores, que rondaba los 12 mil empleados directos. Entre 1991 y 1992, los sucesivos planes de «retiros voluntarios» implementados por los interventores nombrados por el gobierno nacional redujeron en un 50\% el personal ocupado (Rofman y Peñalva, 1995).

Figura 1.

\section{Ubicación geográfica de Villa Constitución}

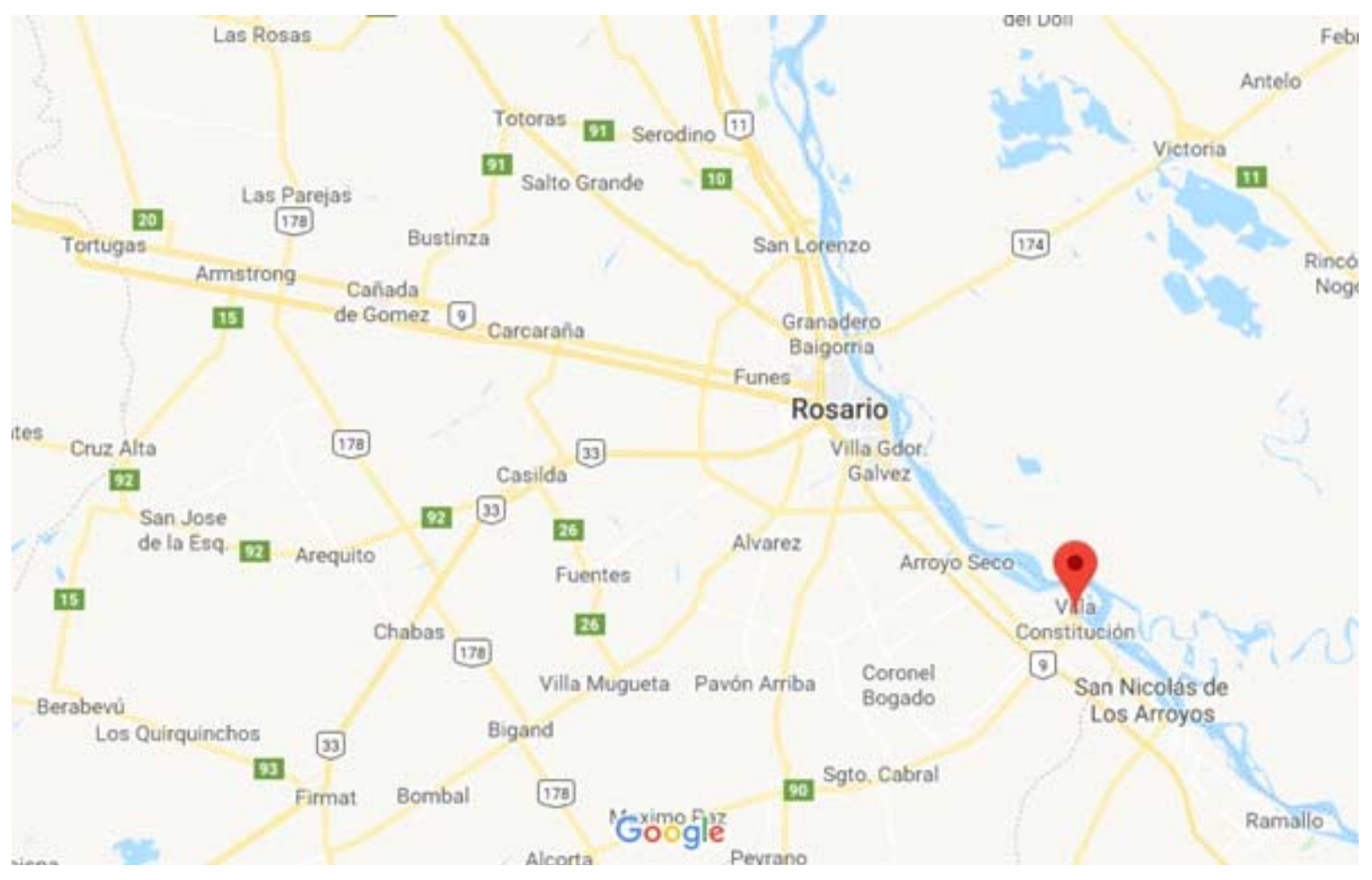

Fuente: google maps. 
Como respuesta ante la situación de desarticulación de las principales fuentes de trabajo del aglomerado San Nicolás-Villa Constitución, y respondiendo a un pedido específico de la dirigencia política villense, en el año 1992, aprovechando la mencionada ley de 1907, Menem decretó la creación de una ZF en esa ciudad santafesina. Tal como lo expresan los fundamentos del decreto 2034, se esperaba que la ZF fuera una herramienta que pudiera mitigar los efectos económicos negativos que el plan económico encarado por el gobierno nacional había producido sobre la actividad siderometalúrgica, mediante la promoción de alternativas productivas y de generación de empleo. En paralelo, el Poder Ejecutivo Nacional habilitó una comisión para la redacción de una propuesta de legislación general sobre ZF en el país que dos años más tarde se convirtió en la Ley 24.331 y reemplazó al decreto 2034 como norma de referencia para la implementación de la ZFS.

El decreto 2034 está fechado el 2 de noviembre de 1992, pero la ZFS fue inaugurada el 12 de abril de 2018. Así, la respuesta que dio el Estado nacional a la crisis de la siderometalurgia en San Nicolás- Villa Constitución tardó 25 años y medio en ponerse en marcha. En ese ínterin pasaron cinco presidentes, siete gobernadores y tres intendentes de Villa Constitución; también ocho ministros de la producción de Santa Fe y cinco presidentes del comité del organismo de administración de la ZFS. Esta importante brecha temporal pone en evidencia la incapacidad del Estado en sus distintos niveles para poner en práctica sus decisiones con la celeridad necesaria para producir una transformación efectiva sobre una realidad problemática ya identificada.

La reaparición en la agenda institucional de las ZF responde al viraje ideológico de principio de los años 90 y a los problemas estructurales que experimentó la matriz productiva Estado-céntrica (Cavarozzi, 1991) desde mediados de los 70. A esto se sumó que durante la totalidad de la década de los 80, la economía nacional no pudo retomar la senda del crecimiento económico.

Una ZF es un dispositivo de comercio exterior cuyo objetivo es el desarrollo y la promoción de las capacidades exportadoras de una economía. Su versión moderna surgió durante la crisis del modo de producción fordista y su reemplazo por un modelo económico basado en las ideas de competitividad y descentralización, que propició la distribución de los eslabones de las cadenas productivas en distintos países, la declinación de los mercados internos y el aumento del comercio internacional. Básicamente, constituyen regímenes de excepción con beneficios impositivos especiales. Existen diferentes tipos: ZF comercial, zona empresarial especial, zona económica especial, zona de servicios financieros, zona de comercio exterior, maquiladoras, zonas de procesamiento de exportaciones (Madoery, 1996).

Las ZF a nivel nacional fueron articuladas para contribuir a la solución de al menos dos problemas más amplios: la baja inserción exportadora de la economía argentina y el creciente nivel de desempleo. En el caso de la ZFS, el énfasis estuvo puesto en la solución del desempleo. Si bien no exclusivamente, la política de ZF en el país y la puesta en marcha de una ZF en Villa Constitución fueron políticas públicas que pretendían dar respuesta a problemáticas muy complejas y generaron un efecto de «apilamiento» (pilling on) (Bardach, 1977), que ocasionó que a los objetivos de una política pública se vayan agregando otros de carácter más general a los que la iniciativa no pudo responder. Así, puede verse cómo los funcionarios públicos nacionales y locales sobreestimaron conceptualmente a las ZFS y pretendieron, a través de ella, dar respuesta a grandes problemas que excedían en mucho a esta política pública.

\section{Dificultades de coordinación durante el proceso de implementación}

Durante las últimas décadas del siglo pasado, los organismos multilaterales de crédito incrementaron su influencia sobre la política económica de los países en desarrollo, promoviendo a escala global una serie de lineamientos político-económicos e institucionales que exigieron la privatización de empresas públicas, la apertura económica y la descentralización y reestructuración de la relación entre el gobierno central y las instancias subnacionales (Orlansky, 2005). En Argentina, la reforma del Estado implicó la desaparición de las instancias formales de planificación (Müller, 2011) y una importante transferencia de funciones hacia las provincias (principalmente educación y salud) y al mercado (por vía de la privatización de empresas prestadoras de bienes y servicios) que, como señala Oszlak (2003, p.15) no fue tanto un achicamiento, sino la «continuidad del Estado por otros medios». Esto implicó una transformación del rol del Estado central, que pasó de ser prestador directo 
de bienes y servicios a un papel de regulador; y, en relación con las instancias subnacionales, de planificador y formulador de políticas y lineamientos generales de gestión.

El diseño de la ley 24.331 es un claro ejemplo de la influencia de estas ideas sobre una política pública. Cao y Vaca (2017) mencionan la existencia de dos modelos de articulación federal que pueden entenderse como dos polos: el federalismo dual y el federalismo cooperativo. El primero, se caracteriza por una división de las injerencias, responsabilidades y funciones; mientras que el otro plantea una articulación cooperativa entre el Estado central y uno o más Estados federados, una acción combinada, donde prima la corresponsabilidad y se busca una articulación sinérgica. La división de atribuciones entre el Estado central y las provincias en la ley 24.331 se ubica más cerca a la idea de un federalismo dual. En este esquema, los Estados subnacionales fueron los encargados de llevar adelante las acciones necesarias para la creación y funcionamiento de las ZF y el gobierno federal cumplió el rol de contralor. Esta dinámica de trabajo resultó problemática y es un caso ilustrativo de la situación que señalan Spiller y Tommasi (2011, p.76) cuando mencionan que «las políticas argentinas no son sólo inestables, sino también pobremente coordinadas entre los niveles de la estructura federal del país y entre los ministerios, secretarías y programas del gobierno nacional».

A continuación, realizamos una síntesis de las etapas que identificamos en el largo proceso de implementación de la ZFS.

\section{Laidea}

En 1991, en Villa Constitución, la posibilidad de crear una ZF fue percibida como un hallazgo, una excepcionalidad que podía ser capitalizada gracias a una ley que había sido letra muerta durante casi un siglo. Por entonces, no existía una legislación general sobre la materia y sólo cuatro territorios estaban potencialmente habilitados para instalar una ZF. La legislación vigente habilitaba la creación de una ZF en la Provincia de Santa Fe desde el año 1907, pero en más de 80 años, la dirigencia provincial nunca había hecho uso de esa atribución. El nuevo contexto de apertura económica de principios de la década de los 90 y la crisis de empleo por la que atravesaba la ciudad hicieron atractiva la idea de la ZF ante los ojos de los dirigentes locales. En este sentido, un grupo de actores ligados al municipio de Villa Constitución comenzó a establecer contactos con el gobierno nacional para lograr que su ciudad sea declarada sede de la ZF que la ley habilitaba para la Provincia de Santa Fe.

La ZF como idea llega a Villa Constitución gracias a un contacto informal. Fue una comunicación personal entre un funcionario municipal y una persona del gobierno nacional o de la empresa ACINDAR (los entrevistados no tienen demasiadas precisiones al respecto), que mencionó o sugirió la idea de armar una ZF en Villa Constitución. Además, fue la buena predisposición de algunos actores locales lo que ocasionó que el proyecto avanzara. Como señalan Sabatier y Weible (2010), estos funcionarios vieron la necesidad y la urgencia de tener que traducir sus objetivos en una política pública antes que otros lo hagan, en este caso, antes que otros actores provinciales, y pusieron manos a la obra rápidamente. Por entonces, no estaba claro quienes podían ser los rivales que potencialmente podrían disputar la ubicación de la ZFS, pero la posibilidad de poder instalarla en Villa Constitución fue interpretada como un golpe de suerte que debía ser aprovechado con celeridad antes de que alguien más lo notara.

Entre 1992 y 1994, la implementación de la ZFS avanzó muy poco, principalmente debido al desconocimiento de los actores sobre la temática (aún no existía ninguna ZF en el país), pero también debido a las dificultades de coordinación con los actores provinciales y nacionales. Por entonces, el país estaba atravesando un proceso de reforma del Estado que reconfiguró las relaciones entre el Estado central y las provincias, hecho el cual ocasionó un disentimiento sobre la urgencia de la iniciativa debido a los compromisos simultáneos que existían en las instancias subnacional y federal, que dejaron a los promotores locales de la ZFS relativamente solos frente al proyecto y con falta de poder para poder llevarlo adelante rápidamente. Esta situación es ilustrativa de algunas de las dificultades que señalan Pressman y Wildavsky (1984) para la coordinación entre los actores implicados en una política pública.

Los actores locales jugaron de manera decidida frente al Estado nacional para conseguir este beneficio, pero se movieron con relativa indiferencia en lo que respecta al Estado provincial. Luego de un año de gestiones 
que avanzaron por canales formales e informales, en noviembre del año 1992, el decreto nacional 2034 estableció la creación de una ZF en la ciudad de Villa Constitución; pero allí también se estableció un diseño de relaciones que otorgó a la Provincia de Santa Fe un rol protagónico en la implementación. A partir de entonces, la implementación de la ZFS estuvo a cargo del MP provincial, el cual debía conformar un órgano administrativo encargado de seleccionar una ubicación para la ZF, realizar el llamado a licitación y luego oficiar de comité de vigilancia sobre las actividades del concesionario. Su presidencia debía estar a cargo de un actor nombrado por el gobierno provincial y, a su vez, la municipalidad de Villa Constitución y las organizaciones representantes de las fuerzas productivas provinciales contaban con un director cada una dentro del ente. La dinámica interna de este organismo no se mostró problemática, pero sí lo fueron sus articulaciones con el ministerio. Esta situación complejizó el esquema de trabajo, ya que los actores políticos provinciales a cargo de la cartera de producción quedaron en el medio de una iniciativa gestionada por los actores locales ante el Estado nacional y de la que ellos no habían participado demasiado hasta el momento. En este marco, dado que sólo puede crearse una ZF por provincia, el tema de la ubicación de la ZFS gravitó sobre las relaciones entre Santa Fe y Villa Constitución. Así, en la práctica vemos cómo dentro del Estado pueden generarse tensiones (Bohoslavsky y Soprano, 2010) a causa de un funcionamiento fragmentado y por la falta de coordinación entre actores de distintos niveles durante la puesta en marcha de una política pública, las cuales conspiran contra el desarrollo efectivo una iniciativa y, a la vez, retroalimentan su fraccionamiento.

\section{La primera implementación}

En 1994, luego de las regulaciones y restricciones introducidas por la ley 24.331, quedó claro que el potencial de las ZF para el desarrollo económico del país era modesto. Sin embargo, a nivel local, la creencia de que la ZFS era la gran oportunidad para resolver los problemas de empleo de la ciudad ya estaba instalada. Así, quienes habían trabajado para ponerla en funcionamiento debían responder por ello en coordinación con un Estado provincial que durante estos primeros años del proceso de implementación no mostró demasiado interés. En este marco, se generaron desconfianzas sobre las intenciones de los actores que debían liderar el proyecto, en el sentido de lo señalado por Pressman y Wildavsky (1984), que conspiraron contra el trabajo colaborativo al interior del área de producción provincial entre actores de Villa Constitución y otros originarios de distintas partes de la provincia.

En términos operativos, la ley 24.331 no introdujo demasiadas variaciones en relación al decreto 2034: era necesario crear un órgano de administración para la ZF; definir una ubicación; llamar a licitación pública para su concesión; que el adjudicatario comenzara con su plan de obras; y, finalmente, que el concesionario comenzara a gestionar la radicación de usuarios en la ZF. Por su parte, la división de atribuciones entre actores locales y provinciales quedaba igual que en el decreto 2034. Este organismo primero ofició de comité de evaluación y selección (el IZFS); luego, una vez adjudicada, el comité de evaluación y selección fue reemplazado por un comité de vigilancia (el EZFS), encargado del control de las actuaciones de la empresa concesionaria. Tanto el comité de evaluación y selección como el comité de vigilancia tuvieron sede en Villa Constitución y, salvo algunas excepciones, las actuaciones del órgano quedaron a cargo de actores políticos y de la producción locales.

Entre 1994 y 1999, se avanzó prácticamente por todo el esquema de implementación, pero existieron algunas situaciones derivadas de cierta desconfianza entre el IZFS y el MP que años más tardes ocasionaron problemas que entorpecieron mucho el desarrollo del esquema de implementación. Esta desconfianza se veía acentuada por la falta de apoyo económico de la provincia, ya que, si bien el IZFS funcionaba en el MP provincial, sus actividades fueron financiadas principalmente con fondos municipales y nacionales. Gracias a estos fondos, los funcionarios del IZFS realizaron un proyecto para la ZFS en colaboración con consultores de la ZF de Shannon (Irlanda) y establecieron una buena dinámica de trabajo con la ZF de La Plata y la Secretaría de Industria nacional, pero existieron algunas complicaciones con la ubicación física de la ZFS derivadas de la falta de coordinación con el gobierno provincial.

En principio, informalmente, se definió una ubicación en un terreno de la empresa ACINDAR, pero producto de un desentendimiento entre la empresa y los actores del MP este terreno no quedó a disposición para la ZFS. Esta situación alimento especulaciones negativas por parte de los actores políticos locales respecto 
del apoyo real que podían obtener de parte de las autoridades del MP. A raíz de esta situación, desde el MP habilitaron la licitación de la ZFS sin una ubicación definida. La empresa adjudicataria compró tierras de una superficie menor (25 hectáreas) a las exigidas por el pliego licitatorio y la Provincia de Santa Fe se comprometió a expropiar terrenos adyacentes para completar la superficie mínima requerida (55 hectáreas), pero en agosto de 1999, los propietarios de las tierras que debían ser expropiadas realizaron un pedido de amparo ante la justicia provincial e interrumpieron el plan de obras que el concesionario había comenzado a desarrollar meses atrás. Esto ocasionó una serie de inconvenientes y demoras que tomó más de 5 años en resolverse. En el medio intervino el gobierno nacional (autoridad de aplicación de la ley 24.331) y el poder judicial provincial.

A causa del lobby desplegado por los propietarios de las tierras a expropiar, el Estado nacional no autorizó el terreno adquirido por la empresa y desde la provincia cambiaron la ubicación de la ZFS. Las demoras ocasionadas y el cambio de a ubicación hicieron que la empresa concesionaria se retirara del proyecto, alegando que la Provincia de Santa Fe había incumplido el contrato de concesión e inició acciones legales que años más tarde se resolvieron por un acuerdo entre las partes. La negativa de los dueños de las tierras ocasionó un efecto dominó que comenzó a desandar el camino realizado durante cinco años y ocasionó la intervención de otros actores que complejizaron en mucho el esquema de implementación, debido, principalmente, siguiendo el argumento de Pressman y Wildavsky (1984), al incremento de la cantidad de actores involucrados que ocasionó un virtual veto de la iniciativa en varias de las nuevas instancias intervinientes.

Sintetizando los acontecimientos de esta etapa, vemos que entre 1994 y 1999, se conformó una red de asuntos (Heclo, 1993) de escala local encabezada por funcionarios municipales que supieron aprovechar algunos vínculos débiles (Granovetter, 1973). El vínculo entre el IZFS y la ZF de La Plata habilitó una buena relación con los funcionarios de la Secretaría de Industria del Ministerio de Economía de la nación; pero no ocurrió lo mismo con las instancias provinciales. Los funcionarios provinciales prestaron apoyo formal al proyecto, pero no avanzar con mayor decisión sobre el tema y tuvieron actitudes semejantes al «juego del apoyo simbólico» o tokenism que señala Bardach (1977), el cual a la larga ocasionó perjuicios sobre la implementación de la ZFS. La actitud especulativa de algunos actores y el efecto del «recurso del diablo» (Sabatier y Weible, 2010) generaron un nivel de desconfianza sobre algunos políticos y económicos de escala provincial y otras localidades. Esta dinámica generó cierto aislamiento político de los actores de Villa Constitución, que ocasionó dificultades a la hora de resolver los problemas judiciales relacionados con las tierras necesarias para continuar la implementación de la ZFS.

\section{Los problemas}

En diciembre del año 1999, asumieron nuevas autoridades a nivel nacional, provincial y municipal. En consecuencia, también cambiaron muchos de los funcionarios encargados de desarrollar la ZFS. El reemplazo de actores al frente del proyecto coincide con el período más problemático del proceso de implementación. Como consecuencia, se desarticuló la red que había funcionado de manera relativamente dinámica en torno a la ZFS hasta el inicio de los problemas judiciales mencionados. Adicionalmente, los nuevos actores a cargo de su implementación se encontraron con un esquema de trabajo que, si bien había avanzado, experimentó un repentino retroceso en su última parte y complejizó aún más la configuración de actores. De este modo, la ZFS pasó de ser una solución para los problemas de Villa Constitución a ser un problema para la dirigencia local y provincial, ya que para su puesta en marcha era necesaria la muy difícil labor de deshacer lo realizado hasta el momento para llegar a cero e iniciar el proceso desde el principio. La situación económica y social argentina por entonces era muy delicada y Santa Fe no era ajena. Esto ocasionó demoras a la hora de resolver los problemas de la ZFS, ya que los funcionarios nacionales y provinciales tenían compromisos simultáneos (Pressman y Wildavsky, 1984) que les hacían disentir sobre la urgencia de la ZFS. Por otro lado, producto del empantanamiento del proceso de implementación, se perdió la creencia de la sociedad local sobre los beneficios colectivos que podría haber traído la ZF, la confianza del mundo de los negocios y el atractivo político del proyecto.

Por entonces, la ZFS era un proyecto empantanado y lleno de problemas. Era riesgoso y poco atractivo para la carrera política de cualquier funcionario porque cualquier acción que se intentara podía llevar al fracaso y dañar el prestigio personal de su responsable. Además, no era un proyecto de primera importancia dentro del MP. En esta situación encontramos una relación inversa con el juego «reputación» de Bardach (1977) en el sentido que señalamos en el apartado teórico. En consecuencia, sólo los actores locales se involucraron activa- 
mente para intentar reflotar la ZFS. Del resto de la dirigencia provincial, a pesar de que mejoraron las relaciones gracias a un diputado provincial oriundo de Villa Constitución con conexiones en la Legislatura provincial, la ZFS sólo obtuvo un apoyo formal (tokenism) (Bardach, 1977).

Recapitulando, vimos cómo entre 1999 y 2003 hubo un avance lento del proceso de implementación. La resolución de los problemas legales necesarios para poder continuar escapó a las posibilidades y atribuciones del EZFS. Tampoco la empresa concesionaria - la cual recordemos que en este contexto solicitó la anulación del contrato - pudo hacer demasiado, ya que pidió retomar las obras, pero la provincia ya había decidido cambiar la ubicación. De este modo, el mayor problema fue que los dos principales actores del proceso, en las etapas finales del esquema, quedaron fuera de juego y a la espera de las decisiones de actores provinciales para los cuales la ZFS era uno más entre otros temas de los que ocuparse. Así, el disentimiento sobre la urgencia de la iniciativa (Pressman y Wildavsky, 1984) generó una situación de virtual paralización de la ZFS, aunque no todo fueron retrocesos en esta etapa, ya que en la Legislatura provincial se sancionó la ley de creación del EZFS y otras relacionados con aspectos técnicos del funcionamiento de la ZFS, que apuntalaron su implementación en lo que toca a cuestiones formales.

\section{La segunda implementación}

En el marco teórico del trabajo caracterizamos al Estado como un espacio polifónico donde se entrecruzan intereses de distintos sectores de la sociedad (Bohoslavsky y Soprano, 2010). En este sentido, a partir de diciembre de 2003, la intervención de la Bolsa de Comercio de Rosario (BCR), que se interesó en el proyecto, apuntaló la implementación de la ZFS. Un cruce de declaraciones públicas en la prensa en tono cordial entre el gobernador Obeid y el titular de la Bolsa de Comercio de Rosario (BCR) ocasionó que la ZFS reingresara a la agenda institucional provincial y contara con la atención y el compromiso del propio gobernador. Además, la inclusión de la BCR introdujo una dinámica diferente a la implementación. Fue la primera vez en la historia del proyecto en la cual una organización de peso del sector privado de escala nacional brindó un apoyo explícito a su materialización y se puso a trabajar para la concreción de la ZFS.

La BCR robusteció la implementación de la ZFS: proveyó asesoramiento técnico, acceso a información, red de contactos y un canal de conversaciones con el ministro y el gobernador. Gracias a este apoyo, se lograron resolver con relativa celeridad los problemas que arrastraba la ZFS desde agosto de 1999 y se pu do alinear a la dirigencia política santafesina, quienes impulsaron una reforma en la ley nacional de ZF para extender los plazos necesarios para su creación.

Durante la gestión del gobernador Obeid, el EZFS modificaron los pliegos con la ayuda de asesores de la BCR. La meta era tener la ZFS adjudicada en diciembre de 2005. Corría marzo de ese año y se pensaba que la meta era posible de cumplir. Había apoyo del gobernador y una coordinación intensa con el MP. Además, los problemas del pasado se habían resuelto. Nunca en los trece años que la idea llevaba en danza habían existido condiciones políticas tan favorables para su concreción. Sin embargo, no todos eran datos positivos, ya que desde el EZFS tuvieron que lidiar con algunos imprevistos. El más importante de ellos fue que, luego de tantos años sin cercar y sin aparente movimiento, sobre la barranca del río Paraná se asentó un grupo de personas en viviendas irregulares, principalmente de pescadores de subsistencia. Un asunto que requirió de la coordinación entre el EZFS, la Municipalidad de Villa Constitución y la Dirección de Vivienda de la provincia.

En paralelo, se realizaron dos llamados a licitación que no tuvieron éxito. En el primero, durante el año 2006, no se vendió ningún pliego; en el segundo, en 2007, ninguno de los dos interesados que adquirieron el pliego realizó una oferta. Sin embargo, a pesar de no haberse podido culminar con éxito el proceso licitatorio, se lograron destrabar las situaciones que tenían paralizada a la ZFS. Vemos así, cómo la complejidad del entramado de actores involucrados en el desarrollo de una política pública puede resolverse cuando existe un apoyo político fuerte que ocasione una ganancia de poder de los implementadores en el sentido que la señalan Pressman y Wildavsky (1984).

En el 2008, excepto por las autoridades locales y los miembros del EZFS, nadie tenía grandes expectativas sobre la realización de esta política pública. Sin embargo, los sucesivos gobiernos provinciales continuaron 
brindando apoyo al proyecto. Por otro lado, los signos políticos nacional y provincial para ese entonces eran diferentes, y el rumbo económico del gobierno nacional no se mostraba compatible con una herramienta económica asociada al liberalismo como son las ZF.

Habían pasado cuatro años sin haber logrado cerrar exitosamente la etapa de licitación. Los plazos se encontraban nuevamente por vencer y una nueva prórroga no parecía ser una opción viable. Para evitar la caída del convenio entre el Estado nacional y el Estado provincial fue el propio EZFS el que comenzó las obras en el terreno. La idea fue que esas obras fueran consideradas a cuenta del futuro concesionario. Los trabajos realizados fueron muy básicos. Lo justo y necesario para cumplir con una formalidad que evitó la cancelación del proyecto. La solución encontrada fue como una especie de atajo entre la complicada trama de reglas y tiempos que el propio Estado había puesto para el cumplimiento de los procedimientos sin dimensionar su complejidad y que, además, el propio Estado dilató excesivamente en más de una oportunidad. El hecho de que las propias disposiciones estatales pasadas y/o de otra dependencia sean las que impiden la acción de otra parte del Estado en el presente nos indica la falta de univocidad que señalamos en el marco teórico (Bohoslavsky y Soprano, 2010).

Finalmente, la ZFS fue adjudicada el 5 de mayo de 2011 al único oferente que había adquirido pliegos, pero hubo que esperar hasta el 26 de enero de 2013 para que la autoridad de aplicación nacional firmara el aval necesario que habilitó la concesión. De este modo, entre el 5 de mayo de 2011 hasta el 26 de enero de 2013, como ya había ocurrido otras veces, la ZFS fue un expediente transitando por reparticiones provinciales y nacionales a la espera de ser firmado por distintas autoridades públicas. Esta situación es un indicador más de cómo la falta de acuerdo sobre la prioridad de un proyecto o la existencia de compromisos simultáneos (Pressman y Wildavsky, 1984) puede ocasionar dilaciones excesivas en la implementación de una política.

El cambio de signo político en el gobierno nacional en diciembre de 2015 también ocasionó dilaciones. La autorización de la licitación y del proceso que llevó al concesionario a la ZFS había sido concedida durante el gobierno de Cristina Fernández, pero aún faltaban autorizaciones de la Aduana para que pudiera comenzar a operar. Desde el gobierno de Macri, se demoró la firma producto de la desconfianza que existía respecto a lo actuado por la anterior gestión. Así, nuevamente, la desconfianza sobre las personas encargadas de liderar un proyecto (Pressman y Wildavsky, 1984) ocasionaron retrasos en su implementación.

Luego de siete años de demoras burocráticas, el 12 de abril de 2018, la ZFS quedó habilitada, aunque con características muy modestas en comparación con el proyecto original. Una síntesis de esta larga etapa de resolución muestra como la decisión política de actores de peso puede destrabar los problemas inherentes a la «complejidad de la acción conjunta», alineando a los actores participantes del proceso de implementación, incluso a aquellos con otras prioridades o intereses. En un contexto de gran actividad, el MP tenía compromisos simultáneos, pero el apoyo de los sucesivos gobernadores al proyecto a partir de 2004 hizo que de todas formas la burocracia provincial se abocara a la implementación de la ZFS. A su vez, pudimos ver cómo la falta de interés de algunos actores y/o las diferencias y desconfianza entre los actores nacionales y subnacionales pueden dilatar los tiempos excesivamente, llevando una iniciativa a una paralización casi total.

\section{Discusión}

La motivación por el análisis de la implementación de la ZFS surgió en base a un interés más amplio de comprender el funcionamiento del Estado, la puesta en práctica de sus políticas públicas y cómo a tal fin se da la articulación entre sus diferentes niveles a través de los actores sociales que «son el Estado el Estado en determinado tiempo y lugar» (Bohoslavsky y Soprano, 2010, p.25).

El diseño de la política nacional de ZF muestra una división del trabajo cercana al modelo de federalismo dual (Cao y Vaca, 2017). Se apoyó en una serie de relaciones interinstitucionales en apariencia no tan complejas, pero en la práctica resultaron poco (co)operativas y dieron paso a una división del trabajo cuya dinámica se mostró ineficiente por la imposibilidad de coordinar agendas institucionales y por la cantidad de pases administrativos por los que tenían que atravesar los expedientes. Esto ocasionó una brecha muy importante entre los 
tiempos de la administración pública, los tiempos de los negocios y la situación sociológica que incentivó la iniciativa. En este sentido, en base a la experiencia analizada, consideramos que una dinámica de relaciones más cercanas a la idea de federalismo cooperativo (Cao y Vaca, 2017) puede ayudar a mitigar los efectos de la desconexión observada entre los tres niveles de gobierno

Vimos entonces cómo en el pasaje del diseño de una política pública a su implementación, la complejidad de la acción conjunta que describen Pressman y Wildavsky (1984) crece y las probabilidades de poder cumplir las metas en los tiempos establecidos decrecen casi tendiendo a cero a medida que pasa mayor cantidad de tiempo y es necesario resolver sucesos contingentes que involucran a un número cada vez mayor de actores; sucesos que, a su vez, alimentan las especulaciones y la posibilidad de divergencia sobre los objetivos originales.

Sin desconocer las limitaciones de este trabajo, confiamos que el caso analizado es un referente empírico donde se hicieron presentes aspectos cuya identificación pueden ser de utilidad en futuras investigaciones para comparar y echar luz sobre la implementación de políticas públicas, principalmente en casos que requieren colaboración o coordinación interjurisdiccional y también para situaciones que contemplan articulaciones entre el sector público y el sector privado. Ante la necesidad de un Estado más eficiente y reflexivo, los estudios sociológicos sobre los factores que afectan a la acción estatal son una condición necesaria para avanzar en esa dirección. De este modo, creemos que pudimos identificar algunas orientaciones prácticas a tener en cuenta para mejorar el diseño y la implementación de políticas públicas:

1. De las ideas de Pressman y Wildavsky (1984) podemos inferir que los diseños y las definiciones de los problemas deben ser simples, ya que la trayectoria ideal de implementación de una iniciativa supone instituciones y actores también ideales, pero los tiempos y las prácticas de las instituciones y los actores reales, a veces, se alejan demasiado del diseño en el papel.

2. En el mismo sentido, consideramos que es conveniente disminuir al menor número posible la inclusión de actores cuya participación no es estrictamente necesaria para los objetivos del proyecto. El desafío para la acción conjunta es poder administrar la tensión entre legitimidad y operatividad, ya que, si bien sumar actores puede dotar de mayor consenso a una política pública, a su vez, puede restarle dinámica en términos operativos.

3. Se desprende también del trabajo de estos autores que intentar poner en marcha una política pública sin los apoyos suficientes es un gasto de tiempo y recursos. Como resultado, se genera un desprestigio hacia la capacidad de acción del propio Estado, que tarde o temprano termina por jugar en contra de sus autoridades presentes o futuras.

4. También, en base al aporte de estos autores, hay que destacar la importancia de la celeridad en el cumplimiento del plan general de implementación, las metas intermedias y en la resolución de situaciones no previstas. Una política pública es la resultante de una situación sociológica específica y, por lo tanto, tendrá sentido para los actores sociales involucrados mientras siga existiendo la situación que estimuló la intervención estatal.

5. Los diseños demasiado rígidos pueden volverse un problema para los propios funcionarios abocados a la implementación. Este tipo de situaciones ocasiona un gran gasto de recursos, tiempo y energías en situaciones que no suman al proyecto y pueden ser evitadas. A su vez, incorporar mecanismos de implementación más flexibles puede ayudar a que los actores involucrados jueguen dentro de las reglas formales establecidas, ya que en algunas ocasiones, las prácticas informales desplegadas tienen la intención de hacer avanzar el proyecto cuando las reglas formales operan como impedimento o demoran su avance.

6. Los funcionarios públicos deben tomar consciencia de la necesidad de construir los problemas en base a una teoría causal del problema (Sabatier y Mazmanian, 1993) y disminuir al mínimo los diseños de políticas basados en su sentido común que los lleve un «apilamiento» de objetivos (Bardach, 1977) que genere expectativas desmedidas sobre los resultados una política pública. 
7. En el sentido de Bohoslavsky y Soprano (2010), es necesario tener en cuenta que las relaciones interinstitucionales son, en última instancia, relaciones entre individuos. Además, son interacciones en donde las capacidades, la legitimidad y las motivaciones de los actores participantes — los privados y los públicos - son de magnitud y naturaleza diferentes, lo cual les da diferente capacidad de acción. Las situaciones son manejadas por funcionarios con intereses materiales y simbólicos asociados a un proyecto político, pero también a sus carreras personales, que ocupan cargos públicos de manera temporal y con un uso limitado de los recursos estatales.

Finalmente, creemos que el estudio de la acción pública es importante por las razones esbozadas hasta aquí, pero principalmente porque el Estado en América Latina rara vez realiza un seguimiento sobre sus intervenciones en la sociedad. Por esta razón, la producción de conocimiento sobre políticas públicas es un eje donde bien puede lograrse una articulación sinérgica entre las ciencias sociales y las instituciones estatales.

\section{Bibliografía}

Bardach, E. (1977). The implementation game. Cambridge, EEUU: MIT Press.

Berman, P. (1993) El estudio de la macro y micro implementación. En L. F. Aguilar Villanueva (comp.). La implementación de las políticas. Ciudad de México, México: Fondo de Cultura Económica.

Bohoslavsky, E. y G. Soprano (2010). «Una evaluación de propuestas para el estudio del Estado en Argentina». En E. Bohoslavsky y G. Soprano (eds.). Un Estado con rostro humano. Funcionarios e instituciones estatales en Argentina (de 1880 a la actualidad). Buenos Aires, Argentina: Prometeo- Universidad Nacional de General Sarmiento.

Cangiano, M. C. (2006). Revisión del pasado y construcción del presente. Los obreros metalúrgicos de Villa Constitución y el menemismo, 1989-1992. Historia Regional, 19 (24), 175-195. Recuperado de http:// historiaregional.org/ojs/index.php/historiaregional/article/view/202/442

Cao, H. y P. Vaca (2017). Federalismo dual y federalismo cooperativo. Documentos de trabajo del CIAP, 17 (2). Recuperado de http://bibliotecadigital.econ.uba.ar/download/docin/docin_ciap_v17_n23

Cavarozzi, M. (1991). Más allá de las transiciones a la democracia en América Latina. Revista de Estudios Políticos (Nueva Época), (74), 85- 111. Recuperado de https://dialnet.unirioja.es/servlet/articulo?codigo=27140

Filippa, V. (2012). Neoliberalismo y flexibilización laboral. El conflicto del '91 en la planta de Acindar de Villa Constitución. Sus efectos en la subjetividad de los trabajadores de base. Historia Regional, 35 (30), 217-236. Recuperado de http://historiaregional.org/ojs/index.php/historiaregional/article/view/49

Giniger, N. (2012). La praxis empresarial en la configuración de una ciudad industrial. El caso de la ciudad siderúrgica Villa Constitución. Intersecciones en Antropología, 13 (2), 439-447. Recuperado de https://ri.conicet.gov.ar/ handle/11336/30940

Granovetter, M. (1973). The strength of weak ties. American Journal of Sociology, 78 (6), 1360-1380. [Traducción: Ma Ángeles García Verdasco]

Heclo, H. (1993). Las redes de asuntos y el poder ejecutivo. En L. F. Aguilar Villanueva (comp.). Problemas públicos y agenda de gobierno. Ciudad de México, México: Fondo de Cultura Económica.

Madoery, O. (1996). Zonas francas. Desarrollo regional y exportaciones. Buenos Aires, Argentina: Ad Hoc. 
Müller, A. (2011). La (no) planificación en la Argentina de los ’90 y los 2000: apuntes para una historia y posibles lecciones para el futuro. Realidad Económica, IADE, (60). Recuperado de http://bibliotecadigital.econ.uba.ar/ econ/collection/docin/document/docin_cespa_025

Orlansky, D. (2005). El Concepto de Desarrollo y las Reformas Estatales: Visiones de los Noventa. Revista Documentos y Aportes en Administración Pública y Gestión Estatal, (6) 41-61. Recuperado de https:// bibliotecavirtual.unl.edu.ar/publicaciones/index.php/DocumentosyAportes/article/view/1198

Oszlak, O. (2003). El mito del Estado mínimo: una década de reforma estatal en Argentina. Desarrollo Económico, 42 (168), 19-543.Recuperado de www.oscaroszlak.org.ar

Pressman, J. y A. Wildavsky (1984). Implementación. Como grandes expectativas concebidas en Washington se frustran en Oakland. Ciudad de México, México: Fondo de Cultura Económica.

Rofman, A. y S. Peñalva (1995). La privatización de SOMISA y su impacto en la producción y el empleo en San Nicolás. Ciclos en la Historia, la Economía y la Sociedad, 5 (8), 55-89. Recuperado de http:// bibliotecadigital.econ.uba.ar/download/ciclos/ciclos_v5_n8_04.pdf

Spiller, P. y M. Tommasi (2011). Un país sin rumbo. ¿Cómo se hacen las políticas públicas en Argentina?. En C. Scartascini, P. Spiller, E. Stein y M. Tommasi (eds.) El juego político en América Latina. Buenos Aires, Argentina: BID. Recuperado de https://publications.iadb.org/es/publicacion/14230/el-juego-politico-en-americalatina-como-se-deciden-las-politicas-publicas

Sabatier, P. A. y Ch. Weible (2010). El marco de las coaliciones promotoras. Innovaciones y clarificaciones. En P. A. Sabatier (ed.). Teorías del proceso de las políticas pública. Buenos Aires, Argentina: Jefatura de Gabinete de Ministros. Recuperado de http://pdfhumanidades.com/sites/default/files/apuntes/66\%20\%20ZAHARIADIS\%20en\%20SABATIER\%20Teo\%20del\%20Proc\%20de\%20las\%20Pol.pdf

Sabatier, P. y D. Mazmanian (1993). La implementación de la política pública: un marco de análisis. En L. F. Aguilar Villanueva (comp.). La implementación de las políticas. Ciudad de México, México: Fondo de Cultura Económica.

Strada, J. (2017). Reconversión productiva y tercerización laboral en la industria: el caso Acindar. H-Industria: revista de historia de la industria, los servicios y las empresas en América Latina, 11 (21), 28-53. Recuperado de http://ojs.econ.uba.ar/index.php/H-ind/article/view/1037 